$16^{\text {th }}$ International Congress of Metrology, 14007 (2013)

DOI: $10.1051 /$ metrology/201314007

(C) Owned by the authors, published by EDP Sciences, 2013

\title{
Hyperspectral imaging: a tool for biological measurements
}

\author{
Marco Pisani ${ }^{1}$, Massimo Zucco ${ }^{1}$, Valentina Caricato ${ }^{1, a}$ and Andrea Egidi ${ }^{1}$ \\ ${ }^{1}$ INRIM Istituto Nazionale di Ricerca Metrologica, 10135 Strada delle Cacce 91, Turin, Italy
}

\begin{abstract}
We present a hyperspectral imaging device (HSI) based on a scanning Fabry-Perot interferometer able to build a hyperspectral cube with high photon collection efficiency (throughput). The device allows to obtain a single image of a sample treated with different fluorescence substances and to unambiguously discriminate them by observing their spectral fingerprint. Furthermore it allows studying biological samples with colorimetric analysis. An experiment carried out with cyanines, fluorescein and quantum dots emitting in the yellow-orange region demonstrates the potentiality of multi-labeled fluorescence microscopy without the use of multiple filter set or dispersive means. The spectra of some biological samples have been computed to demonstrate the validity of the HSI device coupled with an inverted microscope.
\end{abstract}

\section{Introduction}

In biological research and medical diagnostic one of the fundamental goal is to recognize some part of biological samples like membrane, nucleus, chromosomes of a cell and a powerful method to do this is the observation of a particular physical phenomenon called Fluorescence Microscopy (FM) [1]. This effect is observed when a biological sample embedded with a fluorescent substance is illuminated by a light source with a specific spectrum. As result of the interaction between the electromagnetic wave and the fluorescent molecules the target emits light at a longer wavelength. The fluorescent light is filtered in order to stop the exciting light and it is addressed to a camera where the image is recorded. This method has a limit when the sample is made of different targets; in this case different fluorescent substances with different emission spectra should excite each sample. Using a sequence of optical filter, each one for each fluorophore, it is possible to record a sequence of images that can be subsequently merged in a single video. The new image is called multi-labeled sample image. The disadvantage of this method is that it is time consuming and quite expensive [2].

The hyperspectral imaging (HSI) technique can go over this limit. HSI [3] is a powerful measurement tool having manifold applications in different scientific fields such as remote gas sensing, colorimetry, food analysis, skin cancer diagnosis, thermal imaging etc. The HSI device developed at INRIM is basically the combination of a low noise CCD camera and a scanning Fabry-Perot interferometer. This HSI device generates a "hyperspectral cube" where two coordinates are the physical image of the scene, and the third one is the

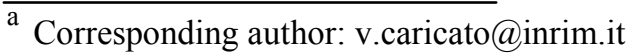

spectral composition of the light hitting each pixel [3]. HSI has been tested in different spectral ranges from the visible [4] to the near IR [5]. The "hyperspectral cube" can be processed in a second step in order to discriminate the different fluorophores through the reconstruction of an RGB image.

The HSI device has been implemented in two different experiments: in the first one the HSI technique was applied to unambiguously discriminate different substances from a multi well sample by fluorescent emission [6]; in the second one the HSI device has been coupled with an inverted microscope in order to obtain preliminary spectra of some biological samples in transmission light. This paves the way to the discrimination of further biological targets by fluorescence microscopy [7].

\section{The experiments}

In the first experiment a multi-well sample with seven different fluorescent substances has been used. Five of them belong to the cyanine dye family: CY 3, CY 3.25 and CY 3.5, characterized by different molar concentrations that will influence the behaviour of the spectra. The sixth substance is fluorescein and the seventh one is a suspension of a CdSe Qdot 560 that shows a wide absorption spectrum in the UV range. The target has been illuminated by a Xenon lamp, with a short pass filter, and excited by a laser at a working wavelength of $405 \mathrm{~nm}$. A mirror addresses the fluorescence light emitted by the sample to the HSI device through a "longpass" filter. While the Fabry-Perot cavity scans the optical path delay, a camera (PCO Pixelfly) records a video that has been subsequently analyzed with a Fourier 
Transform based algorithm in order to obtain the hyperspectral cube [3-5].

Figure 1. The experimental layout with the HSI device

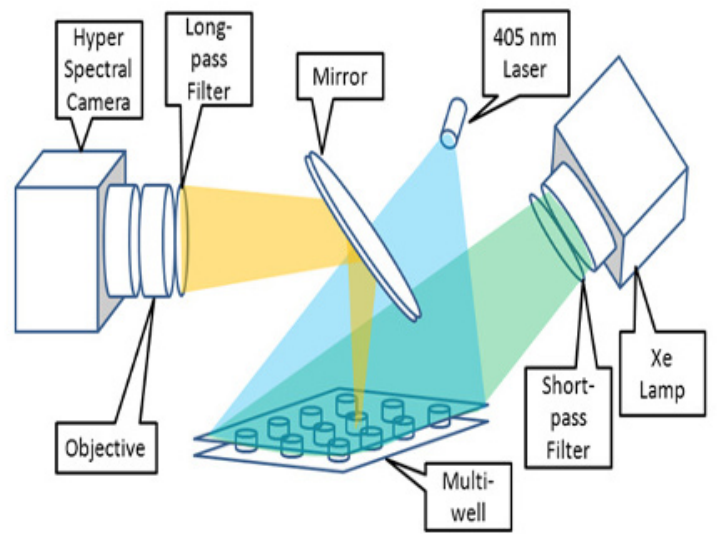

In the second experiment we have used a Zeiss microscope that works with transmitted and fluorescence lights coupled with our HSI device and the PCO Pixelfly camera. A biological sample is directly lighted by a lamp on the top of the microscope and on the bottom there is an objective that collects the light itself. A system of internal optics addresses the light both on the eyepieces of the microscope and on one side of the microscope where a camera adapter is mounted. For our purpose we have removed this adapter and we have fixed a new lens with a focal distance $\mathrm{f}=100 \mathrm{~mm}$. On this side of the microscope an image of the sample is visible. Then, considering the depth of our HSI device, we have fixed the Fabry-Perot in order to have the image in the center of the cavity. On the back of the HSI we have fixed a relay lens for the purpose of extending the system and inverting the image. Finally, a band pass optical filter that transmits wavelength in the range between $400 \mathrm{~nm}$ and $720 \mathrm{~nm}$ is fixed in the back of the relay itself. Then the PCO Pixelfly camera is placed on the back of the relay lens approximately in the new focal plane. We have added a laser at $405 \mathrm{~nm}$ as a reference for the calibration of the optical path length of the Fabry-Perot cavity during the scanning. This laser works in pulsed mode in order to have a precise sequence of alternates frames: one with the reference laser and the sample and another one with the sample alone. This method allows reconstructing in a single video the interferograms both of the laser and of the reflected light of the biological sample during the scanning of the cavity. The record of the video has been described in the first experiment. Figure 2 shows a layout of the second experiment.
Figure 2. The experimental layout with HSI device and the microscope.

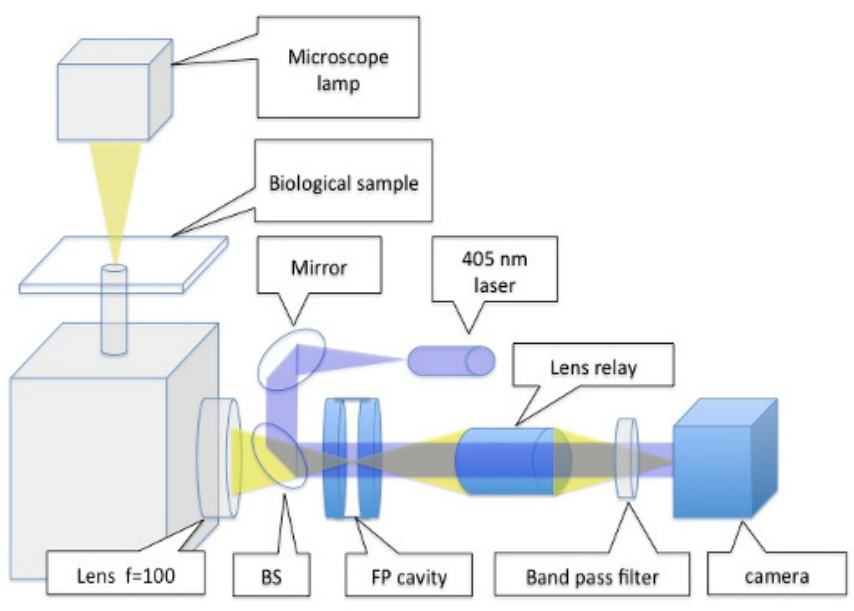

\section{Results and discussion}

\subsection{First experiment}

Figure 3 refers to one frame of the video of $300 \times 300$ pixels where the fluorescent substances are in wells A2, B1, B2, B3, C1, C2 and C3. Figure 4 shows the normalized spectra of the seven substances in the sample. All the spectra are rescaled in order to be comparable. Cyanine are typically characterized by spectra with emission double peaks, whereas Qdot spectrum has its maximum at $560 \mathrm{~nm}$ close to the cutting wavelength of the long pass filter $(575 \mathrm{~nm})$.

Figure 3. A frame of the video with seven different fluorescent substances to be analyzed.

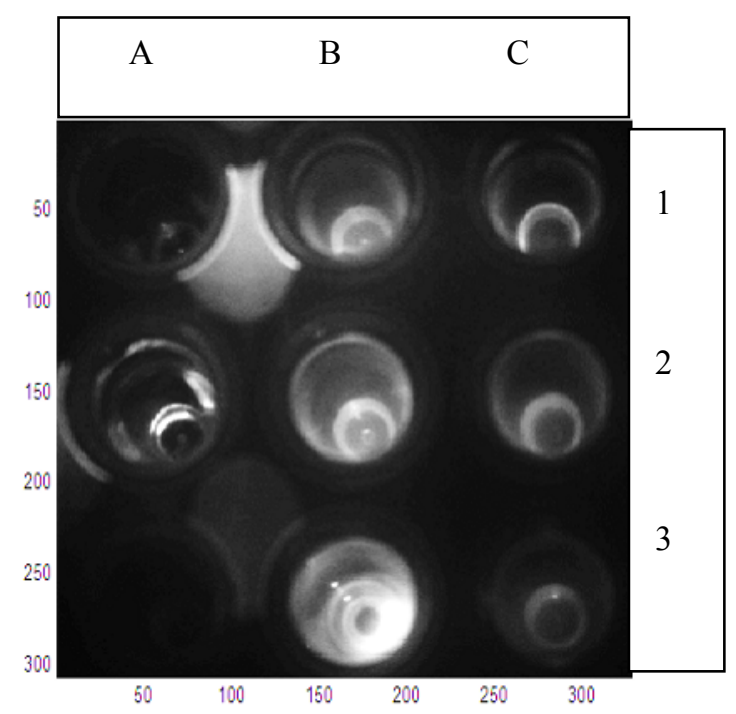


Figure 4. The spectra calculated for a subset of pixels for each well of the sample in Figure 3.

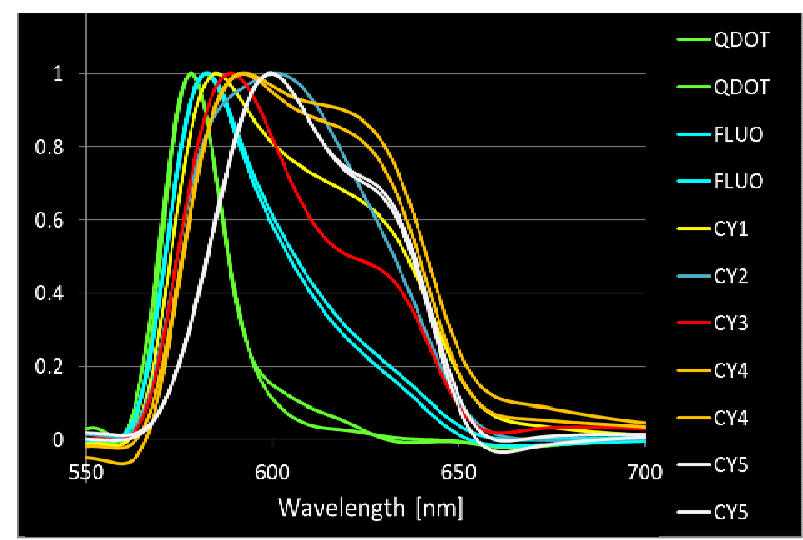

The reference spectra $S_{R}$ of Figure 4 have been computed on a subset of pixels formed by about 20 pixels in the central region of each well. Each of the seven spectra $S_{R}$ defines the reference for the subsequent discrimination of all the different substances in the image. For each pixel spectrum $S_{M}$, the seven distances $d_{R}$ with respect to the seven substance spectra $S_{R}$ have been calculated with the Euclidean distance:

$$
d_{R}=\sqrt{\sum_{\lambda_{i}=550 n m}^{700 n m}\left(S_{M}\left(\lambda_{i}\right)-S_{R}\left(\lambda_{i}\right)\right)^{2}}
$$

where $S_{M}\left(\lambda_{i}\right)$ and $S_{R}\left(\lambda_{i}\right)$ are the wavelength bins. Finally, the pixel is assigned to the class having the shortest distance. The result of the discrimination is presented in the false color image in Figure 5 where the saturated pixel and the pixel belonging to the container base are set to black.

Figure 5. False color image with the classification of each pixel to one of the seven substances.

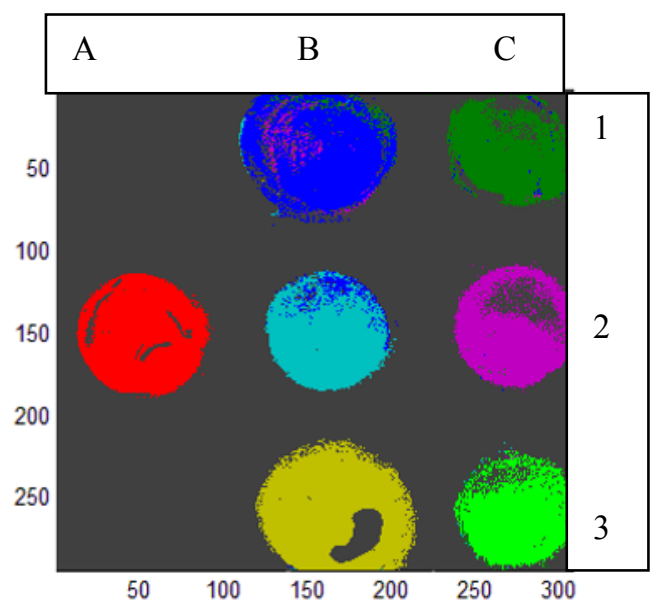

\subsection{Second experiment}

In the second experiment the HSI device has been coupled with a microscope in order to extract the spectra of some biological samples. In Figure 6 a frame of a pine wood of dimension $1400 \times 1000$ pixels has been represented. The sample has two dyes substances (reddish and bluish) visible by transmission light through the microscope eyepieces.

Figure 6. A frame of a pine wood biological sample

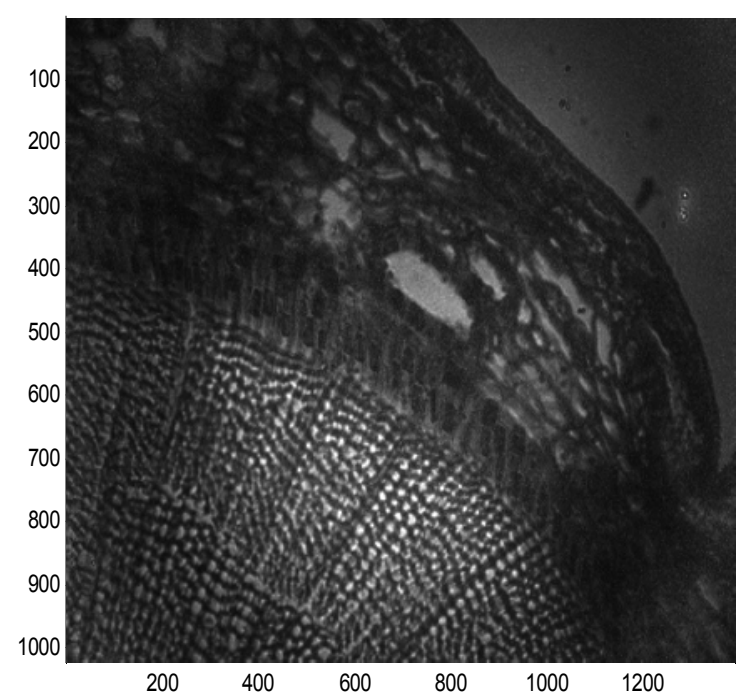

We have selected three different regions of the samples of approximately $30 \times 45$ pixels to discriminate the precence of the different dyes. In this way a dedicated labview software extracts the mean spectra starting from the interferogram of the recorded video and computes the Fast Fourier Transform (FFT). In Figure 7 we show a coloured picture of the sample where three regions with different spectra have been selected. One of the region, the white one, has been used as the white reference to normalize the spectra in the other two regions.

Figure 7. A coloured picture of the pine wood sample with the selection of the regions with different dyes.

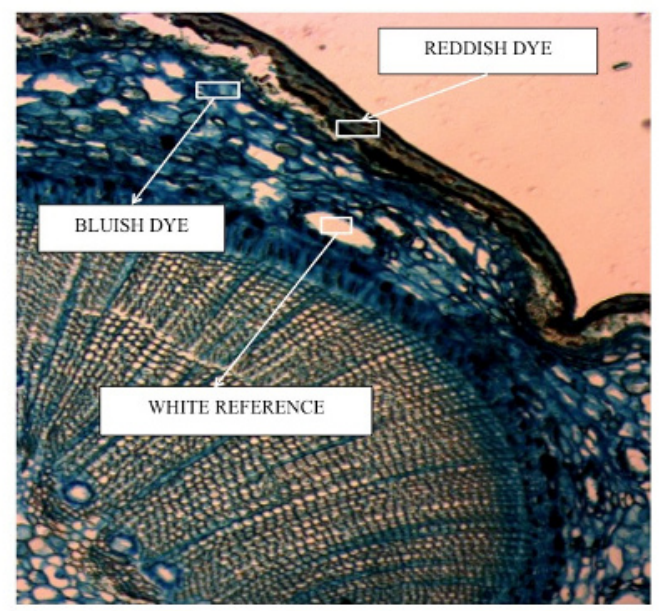

In Figure 8 the abundance of different dyes is visible in the resulting spectra normalized with a white reference. 
Figure 8. The normalized spectra showing different concentration of reddish and bluish dyes.

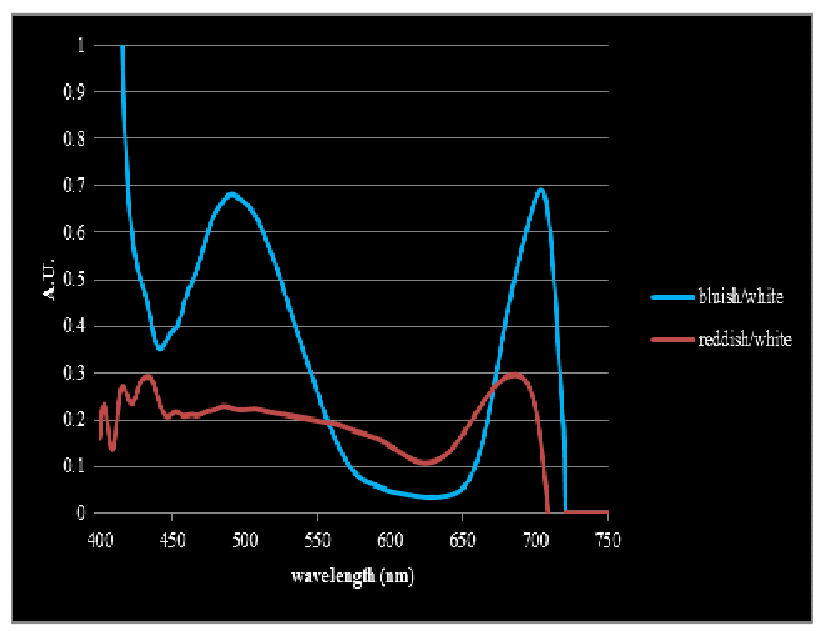

Looking at the normalized spectra in Figure 8 we can see that it is possible to easily recognize the reddish dye, whereas the bluish spectra shows the effect of both the dyes. The sharp slope around $420 \mathrm{~nm}$ is due to the normalization around one of the limit of the band pass optical filter.

\section{Conclusion}

In this work we have demonstrated, with two correlated experiments, how to discriminate the spectra of different fluorescent substances used in bio-science with an innovative HSI device developed at INRIM that allows to record different spectra in a single video. This paves the way to the development of a new fluorescence instrument made of the HSI device combined with a classical microscope. With these two optical instruments we have demonstrate the possibility to observe a pine wood biological sample in transmission light and this will lead to the observation of fast dynamic processes of live samples, reducing the risk of light-damage, typical of fluorescence microscopy.

\section{Acknowledgments}

The authors would like to acknowledge Leonardo Mortati and Carla Divieto from the Biometrology Group of INRIM for their help in the conception and preparation of the samples used in the experiment.

\section{References}

1. Y. Hiraoka, T. Shimi, T. Haraguchi, Cell Structure and Function, 27, 367 (2002)

2. Y. Garini, I.T. Young, G. McNamara, Cytometry Part A, 69A, 735 (2006)

3. M.Q.Pisani and M.E.Zucco, Fourier Transforms Approach to scientific principles, (Goran Nicolic, book chapter $n^{\circ}$ 21-ISBN 978-953-307-231-9, 2011)

4. M.Pisani, M.Zucco, Opt. Exp.,17, 8319 (2009)
5. M.Pisani, P.Bianco and M. Zucco, App. Phy. B: Lasers and Optics, 108, 231 (2012)

6. E. Schröck, S. du Manoir, T. Veldman, B. Schoell, J. Wienberg, M. A. Ferguson-Smith, Y. Ning, D. H. Ledbetter, I. Bar-Am, D. Soenksen, Y. Garini, T. Ried, Science, 273, 494 (1996)

7. N. Ben-Yosef, R. A. Buckwald, D. Cabib, Z. Malik, Spectral bio-imaging methods for biological research, medical diagnostics and therapy (US5991028 A, Nov 23, 1999) 